\title{
Nano-Se crosstalks with nano-DOX/FU to selectively hack hepatic cancer cells and spare normal cells healthy: A mechanism-based study
}

\author{
Aziza B. Shalby, Ahmed A. Abd-Rabou, Hanaa H. Ahmed* \\ Hormones Department, Medical Research Division, National Research Centre, Cairo, Egypt.
}

\begin{tabular}{l} 
ARTICLE INFO \\
\hline Article history: \\
Received on: 10/04/2017 \\
Accepted on: 22/05/2017 \\
Available online: $30 / 08 / 2017$ \\
\hline Key words: \\
Nano-Se, nano-FU, nano- \\
DOX, HepG2 cell line, tumor \\
inhibition.
\end{tabular}

Accepted on: 22/05/2017

Available online: 30/08/2017

Nano-Se, nano-FU, nanoinhibition. \section{(1)}


Moreover, anticancer potential of Se can be manifested by two mechanisms; the induction of apoptosis and cell-cycle arrest. Both mechanisms may be shared together in modulation of the redox reactions, the detoxification of carcinogens, and the inhibition of angiogenesis (Liang et al., 2016). Other mechanisms have been proposed as Se plays a role in the regulation of other axis related to stress response, protein synthesis, cell migration and cell cycle, including p38 MAPK, $\mathrm{p} 53$, p70S6K and protein ubiquitination signaling pathways (Wang et al., 2016).

The nanoparticles of the FDA approved biodegradable/ biocompatible polymer, Poly D-L-lactide-co-glycolide (PLGA), is widely used for the delivery of various chemotherapeutic candidates to the target site. However, rapid opsonization by cells of the phagocytic system is a major limitation for achieving effective drug targeting to the site of action by PLGA nanoformulation. Thus, to maximize the therapeutic benefits of drug loaded nanoparticles (NPS), they should be able to evade the reticulo-endothelial system (RES) (Gref et al., 1994). Surface coating by hydrophilic polymers such as chitosan (CS) and polyethylene glycol (PEG) were used to curb the phagocytic effects and to enhance the longevity of the nanoformulations. Chitosan is a biodegradable, biocompatible polymer with low toxicity and ability to enhance the penetration of macro-molecules across mucosal surfaces (Illum, 1998; Hu et al., 2008; Parveen et al., 2010). Hydrophilic poly (ethylene glycol) (PEG) was introduced as an additional coating polymer to form PEG coated (PLGA-CS-PEG) nanoformulations; because, the chemical modification of CS with PEG not only improves the biocompatibility of CS (Zhang et al., 2001; Abd-Rabou and Ahmed, 2017), but also reduces the adsorption of circulating plasma proteins onto the material surface (Amiji, 1997).

The current study aimed at investigating the impact of the active cross taking between nano-Se and DOX/FU-loaded PLGA-CS-PEG nanoformulations on hepatic cancer (HepG2) and normal (BHK-21) cell line with special focus on mitochondrialbased cytotoxicity and cell cycle. Besides, nitric oxide (NO) and malondialdehyde (MDA) as stress markers, as well as glucose uptake and Zinc activation will be quantitatively tested upon Se, DOX (doxorubicin), and FU (5-fluorouracil) nanoformulation individual or in combination using a range of treatment doses.

\section{MATERIALS AND METHODS}

\section{Preparation of Nano-Se}

One $\mathrm{ml}$ of $25 \mathrm{mM}$ sodium selenite was mixed with $4 \mathrm{ml}$ of $25 \mathrm{mM}$ glutathione (GSH) containing $20 \mathrm{mg}$ bovine serum albumin (BSA). The $\mathrm{pH}$ of the mixture was adjusted to 7.2 with 1.0 M sodium hydroxide, instantly forming nano red elemental Se and oxidized glutathione (GSSG).

The red solution was dialyzed against double distilled water for $96 \mathrm{~h}$, the water changed every $24 \mathrm{~h}$ to separate GSSG from the Nano-Se. The final solution containing nano-Se and BSA was lyophilised and stored at room temperature.

\section{Transmission electron microscopic (TEM)}

Particle morphology and size of nano-Se were examined by transmission electron microscopy (TEM, Philips CM-10, FEI Inc., Hillsboro, OR, USA). $100 \mu \mathrm{g} / \mathrm{ml}$ of the nano-Se suspensions (pre- and post-dialysis) were dropped into Formvar-coated copper grids, and after complete drying, the samples were stained using $2 \%$ w/v uranyl acetate (Electron Microscopy Services, Ft. Washington, PA). Image capture and analysis was done using Digital Micrograph and Soft Imaging Viewer Software.

\section{Preparation of PLGA-CS-PEG nano-void}

Poly D, L-lactide-co-glycolide (PLGA) nanoparticles were prepared by oil-in-water $(\mathrm{O} / \mathrm{W})$ single emulsion solvent evaporation method (Parveen and Sahoo, 2011), with slight modifications. Briefly, $100 \mathrm{mg}$ of PLGA polymer was dissolved in $3 \mathrm{ml}$ of chloroform to form a primary emulsion which was further emulsified in an aqueous polyvinyl alcohol (PVA) solution $(12 \mathrm{ml}$, $2 \% \mathrm{w} / \mathrm{v}$ ) to form an oil-in-water emulsion using a micro-tip probe sonicator (VC 505, Vibracell Sonics, Newton, USA) set at 55W of energy output for $2 \mathrm{~min}$ over an ice bath. For the preparation of polyethylene glycol/ chitosan-blended PLGA(PLGA-CS-PEG) nano-void, $12 \% \mathrm{w} / \mathrm{w}$ of CS solution in 1\%glacial acetic acid and $5 \% \mathrm{w} / \mathrm{w}$ of $2 \mathrm{kD}$ PEG were was added to the aqueous PVA solution prior to emulsification with the PLGA polymer to get PLGA-CS-PEGnano-void.

The emulsion was stirred $8 \mathrm{~h}$ for the evaporation of the organic solvent. Excess amount of PVA was removed next day by ultracentrifugation at $50,602 \times \mathrm{g}$ at $4{ }^{\circ} \mathrm{C}$ for $20 \mathrm{~min}$ (Sorvall Ultra speed Centrifuge, Kendro, USA) followed by washing twice with double distilled water.

\section{Preparation of DOX and 5FU-loaded PLGA-CS-PEG}

For therapeutic applications, Doxorubicin (DOX) and 5Fluorouracil (5FU)-encapsulated PLGA-CS-PEG nanoparticles (Nano-DOX and Nano-FU) were prepared in a similar way as mentioned. $5 \mathrm{mg} / \mathrm{ml}$ of DOX and $5 \mathrm{FU}$ were added to the polymer solution prior to emulsification.

\section{Particle size analysis and zeta potential measurement}

Particle size and zeta potential of the PLGA-CS-PEG nanoparticles was determined by photon correlation spectroscopy (PCS) using a Zeta Sizer (Nano ZS, Malvern Instruments, UK), with a red laser of wave length $\lambda_{0}=633 \mathrm{~nm}(\mathrm{He}-\mathrm{Ne}, 4.0 \mathrm{Mw})$. For size measurements, $1 \mathrm{mg}$ of the nanoparticles was dissolved in $1 \mathrm{ml}$ of water which was further diluted 10 times with water and measured for a minimum of $120 \mathrm{~s}$. Similarly for zeta potential measurements, the samples were placed in an electrophoretic cell, where a potential of $\pm 150 \mathrm{mV}$ was established. Nanoparticles were maintained at a constant temperature of $25.0 \pm 0.1{ }^{\circ} \mathrm{C}$.

\section{In-vitro studies}

Cell culture, maintenance, and sub-culture

Human hepatoma cells (HepG2) and normal kidney cells (BHK-21) were purchased from American Type Culture 
Collection (ATCC, USA) and cultured using a Roswell Park Memorial Institute (RPMI) 1640 medium and Hanks' Modified Eagle Medium (Hanks' MEM), respectively, with 10\% fetal bovine serum (FBS), $1 \%$ penicillin/streptomycin (P/S), and $1 \% \mathrm{~L}$ glutamine obtained from Life Technologies, Gibco (Grand Island, NY).

Cells were cultured in $5 \% \mathrm{CO}_{2}$ at $37^{\circ} \mathrm{C}$ and then treated with $0.25 \%(\mathrm{w} / \mathrm{v})$ trypsin/EDTA to affect cell release from the culture flask. After washing the cells with phosphate buffered saline (PBS), cells were suspended in the media.

\section{Drug Screening and cytotoxicity Drug grouping}

There are 10 drug groups were applied on HepG2/BHk21 cells to test their mitochondrial function and in turn cell viability up on treatment. Se and nano-S with different concentrations $(0,2,4,6,8,10,30$, and $50 \mu \mathrm{M})$ were applied on HepG2/ BHk-21 cells. Moreover, FU, DOX, nano-FU, and nanoDOX with different concentrations $(0,10,20,30,40$, and $50 \mu \mathrm{M})$ were tested up on HepG2/ BHk-21 cells. On the other hand, $50 \mu \mathrm{M}$ FU plus serial doses of Se, $50 \mu \mathrm{M}$ DOX plus serial doses of Se, 50 $\mu \mathrm{M}$ nano-FU plus serial doses of nano-Se, and $50 \mu \mathrm{M}$ nano-DOX plus serial doses of nano-Se (i.e. $0+0,50+2,50+4,50+6,50+8$, $50+10,50+30$, and $50+50 \mu \mathrm{M}$ ) were applied on HepG2/BHk-21 cells.

\section{3-(4, 5-dimethylthiazol-2-yl)-2,5-diphenyltetrazolium bromide (MTT) assay}

All drug groups were evaluated by MTT assay using HepG2/BHk-21 cells. Briefly, the cells were cultured in 96-well plates at a density of $5 \times 10^{3}$ cells/well. All drugs with their described concentrations were added in RPMI-1640 (HepG2 cells) and Hanks' MEM (BHK-21 cells). Culture media with nano-void and without were added as controls for the drug-loaded nanoformulations and their free counterparts. After a day incubation, MTT (Sigma) dissolved in PBS was added to each well at a final concentration of $5 \mathrm{mg} / \mathrm{ml}$, and the samples were incubated at $37^{\circ} \mathrm{C}$ for $4 \mathrm{~h}$. Water-insoluble dark blue formazan crystals that formed during MTT cleavage in actively metabolizing cells were then dissolved in dimethyl sulfoxide (DMSO). Absorbance was measured at $455 \mathrm{~nm}$, using a microplate reader (BMG Labtech, Germany). The cell viability (\%) was calculated and compared with the controls.

\section{Glucose uptake measurement}

Glucose level accumulation in RPMI-1640 medium of HepG2 cells and Hanks' MEM medium of BHK-21 cells were measured up on different nano-treatments and their free counterparts using glucose detection kit (Spectrum Diagnostics). Briefly, the cells were cultured in 96-well plates at a density of $1 \times 10^{4}$ cells/well. In the second day, $5 \mathrm{mM}$ of glucose (Sigma) and different nano-treatments and their free counterparts were added in the media after $2 \mathrm{~h}$ of cells starvation. $10 \mu \mathrm{M}$ of all $\mathrm{Se}$ formulations and $50 \mu \mathrm{M}$ of all DOX and 5FU formulations were used in this experiment. Absorbance was measured at 450nm using the microplate reader (BMG Labtech, Germany) after $10 \mathrm{~min}$ incubation with the glucose detector. The glucose levels in $\mathrm{mg} / \mathrm{dL}$ was calculated and compared with the untreated and nano-void controls.

\section{MDA assay}

Lipid peroxidation refers to the oxidative degradation of lipids. In this process free radicals take electrons from the lipids in cell membranes, resulting in HepG2 and BHK-21cells damage. Quantification of lipid peroxidation is essential to assess oxidative stress in pathophysiological processes. The end product of lipid peroxidation is malondialdehyde (MDA). Measuring the end product of lipid peroxidation is one of the most widely accepted assays for oxidative damage. Lipid peroxidation colorimetric assay kit (ab118970) is a sensitive detection tool of MDA. Briefly, the cells were cultured in 96-well plates at a density of $1 \times 104$ cells/well.

In the second day, different nano-treatments and their free counter parts were added in the media. $10 \mu \mathrm{M}$ of all Se formulations and $50 \mu \mathrm{M}$ of all DOX and $5 \mathrm{FU}$ formulations were used in this experiment. The free MDA present in the sample was reacted with Thiobarbituric Acid (TBA) to generate a MDA-TBA adduct,which can be easily quantified colorimetrically at OD 532 $\mathrm{nm}$ using the microplate reader (BMG Labtech, Germany).

\section{NO measurement}

Nitric oxide (NO) plays an important role in vascular regulation, immune response, and apoptosis. $\mathrm{NO}$ is rapidly oxidized to nitrite and nitrate which are used to quantify NO production. Briefly, the cells were cultured in 96-well plates at a density of $1 \times 10^{4}$ cells/well. In the second day, different nanotreatments and their free counterparts were added in the media. 10 $\mu \mathrm{M}$ of all Se formulations and $50 \mu \mathrm{M}$ of all DOX and $5 \mathrm{FU}$ formulations were used in this experiment. NO assay colorimetric kit (ab 65328) was used to measure the total nitrate/nitrite in a simple two-step process. Nitrate reductase was firstly used to converts nitrate to nitrite.

Then, Griess reagent was used to converts nitrite to a deep purple azo compound. The amount of the azo chromophore accurately reflected nitric oxide amount in the samples. Finally, optical density was measured at OD $540 \mathrm{~nm}$ using the microplate reader (BMG Labtech, Germany).

\section{Zinc levels detection}

Zinc (Zn), a metallic chemical element, is an essential mineral of great biological significance, because many enzymes require it as an essential cofactor, mediates signal transduction, gene-expression, and apoptosis. Zinc was quantified using a convenient colorimetric assay (ab102507). Briefly, the cells were cultured in 96-well plates at a density of $1 \times 10^{4}$ cells/well. In the second day, different nano-treatments and their free counterparts were added in the media. $10 \mu \mathrm{M}$ of all Se formulations and $50 \mu \mathrm{M}$ of all DOX and 5FU formulations were used in this experiment. 
Practically, $\mathrm{Zn}$ was bound to a ligand and the colored product was then detected at OD560 $\mathrm{nm}$ using the microplate reader (BMG Labtech, Germany).

\section{Cell cycle assay}

G0/G1, S, and G2/M phases of cell cycle were evaluated by measuring the cellular uptake of PI (Sigma-Aldrich) by flow cytometer instrument (Beckman Coulter, USA). Briefly, the cells were cultured in 6-well plates at a density of $1 \times 10^{6}$ cells/well. In the second day, $10 \mu \mathrm{M}$ of Nano-Se and $10 \mu \mathrm{M}$ of Nano-Se plus 50 $\mu \mathrm{M}$ of nano-DOX were applied on HepG2 and BHK-21 cells and tested against the untreated cells.

\section{Statistical analysis}

All assays were repeated three times. Comparisons between nano-formulations and their free counterparts versus controls were made using a two-tailed Student's t test, and values of $\mathrm{P}<0.05$ were considered statistically significant.

\section{RESULT AND DISCUSSION}

\section{Se and nano-Se induce HepG2 and BHK-21 cell death}

Fig. 1 shows the nano-Se TEM images. The morphology of nano-Se is spherical as shown in (Fig. 1a) and its size is around $70 \mathrm{~nm}$ as shown in (Fig. 1b). Fig. 2 shows PLGA nanoparticles with size (201 nm) as shown in (Fig. 2a) and zeta potential (+7.26 $\mathrm{mV}$ ) as shown in (Fig. 2b). The cytotoxicities of Se and nano-Se were tested against hepatoma cell line (HepG2) and normal kidney cells (BHK-21) at different concentrations (0, 2, 4, 6, 8, 10, 30, and $50 \mu \mathrm{M}$ ) using MTT assay. Data illustrated in (Fig. 3) shows the percentage of viability of HepG2/ BHK-21 cells after $24 \mathrm{~h}$ from treatments versus controls. The results revealed a dose dependent statistical decrease $(\mathrm{P}<0.01)$ in cell viability of HepG2/ BHK-21 cells.

Also, there were no significant differences between both cell lines; where Se had the highest cytotoxic effects at $50 \mu \mathrm{M}$ with $56.5 \%$ (Fig. 3a) and $58.9 \%$ (Fig. 3b) cell viabilities, respectively, while nano-Se had the highest cytotoxic effects at 50 $\mu \mathrm{M}$ with $57.8 \%$ (Fig. 3a) and $68.2 \%$ (Fig. 3b) cell viabilities, respectively, at $24 \mathrm{~h}$.

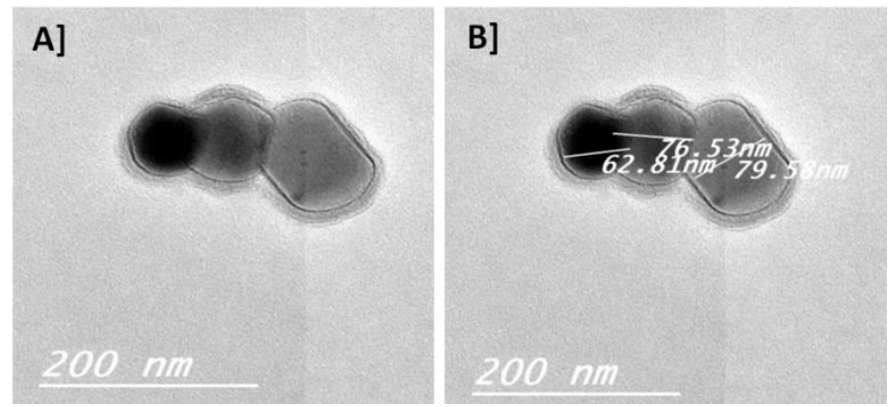

Fig. 1: nano-Se TEM images. Nano-Se morphology (Fig. 1A) and size (Fig. 1B) were represented. A) The TEM images of three rounded nanoparticles were illustrated. B) The sizes $(62.8 \mathrm{~nm}, 76.5 \mathrm{~nm}$, and $79.5 \mathrm{~nm})$ of these three nanoparticles were measured using TEM system software (TEM, Philips CM10, FEI Inc., Hillsboro, OR, USA).
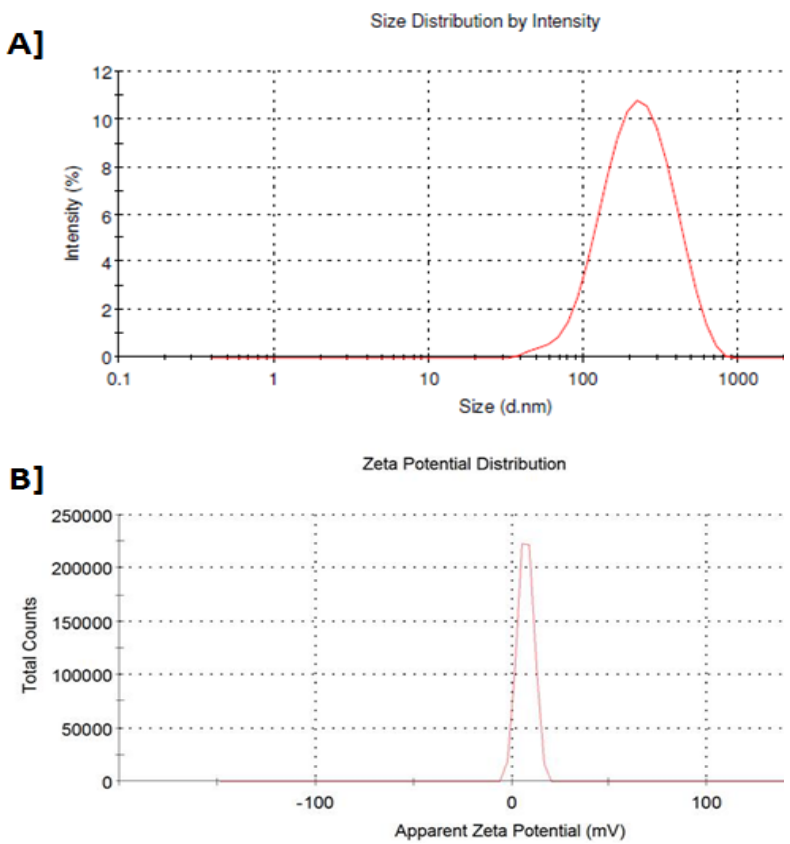

Fig. 2: PLGA-CS-PEG nanoparticles were characterized using Malvern Zeta Sizer. Size $(201 \mathrm{~nm})$ (Fig. 2A) and zeta potential $(+7.26 \mathrm{mV})$ (Fig. 2B) of PLGA-CS-PEG nanoparticles were measured. Nanoparticles was determined by photon correlation spectroscopy (PCS) using a Zeta Sizer (Nano ZS, Malvern Instruments, UK), with a red laser of wave length $\lambda_{0}=633 \mathrm{~nm}(\mathrm{He}-$ $\mathrm{Ne}, 4.0 \mathrm{Mw}$ ). Nanoparticles were maintained at a constant temperature of $25.0 \pm 0.1^{\circ} \mathrm{C}$

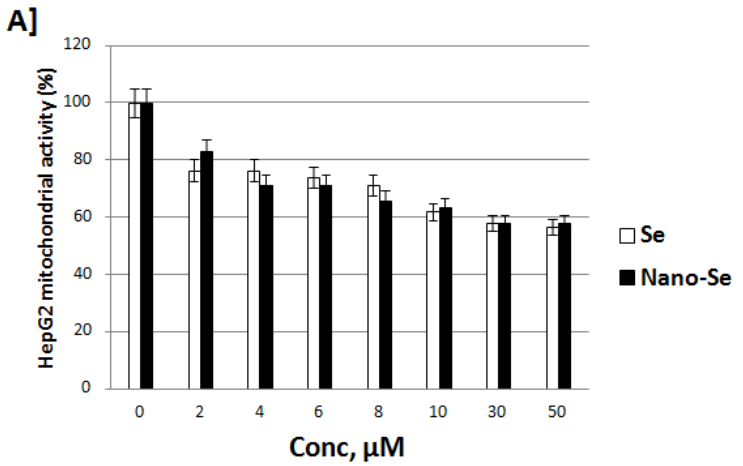

B]

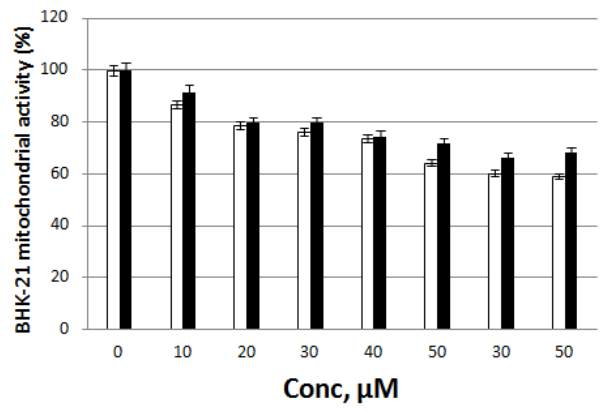

Fig. 3: The cytotoxic-based mitochondrial dysfunction of HepG2 (Fig. 3A) and BHk-21 (Fig. 3B) cells upon Se and nano-Se. Cytotoxicity of Se and nano-Se was evaluated by MTT assay using HepG2/BHk-21 cells. Absorbance was measured at $455 \mathrm{~nm}$, using a microplate reader (BMG Labtech, Germany). The cell viability (\%) was calculated and compared with the controls. Three independent runs $(n=3)$ were performed for each experiment and represented as mean and standard deviation (SD) bars. The results revealed a dose dependent statistical decrease $(\mathrm{P}<0.01)$ in cell viability of HepG2/ BHK-21 cells. 
A]

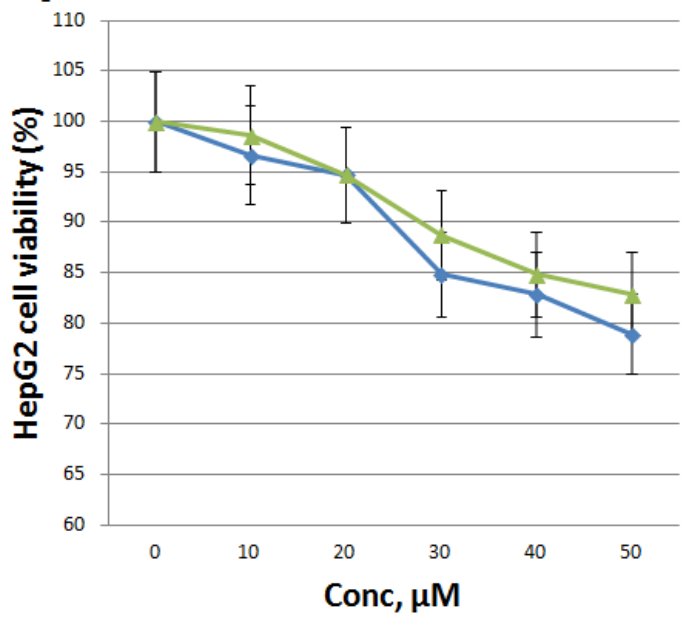

B]

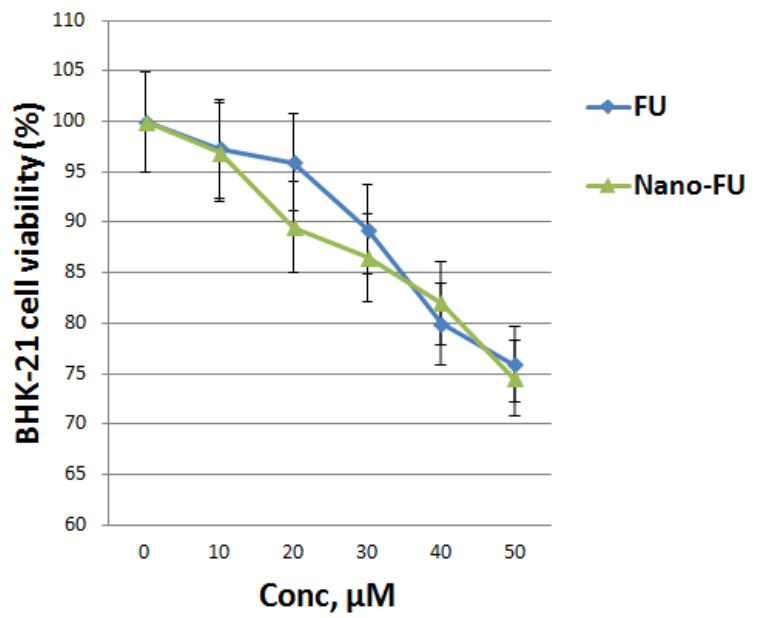

Fig. 4: Nano-FU and its free counterpart induce cytotoxicity in both HepG2 (Fig. 4A) and BHk-21 (Fig. 4B) cells. Cytotoxicity of FU and Nano-FU was evaluated by MTT assay using HepG2/BHk-21 cells. Absorbance was measured at $455 \mathrm{~nm}$, using a microplate reader (BMG Labtech, Germany). The cell viability $(\%)$ was calculated and compared with the controls. Three independent runs $(\mathrm{n}=3)$ were performed for each experiment and represented as mean and standard deviation (SD) bars. The results revealed a dose dependent statistical decrease $(\mathrm{P}<0.05)$ in cell viability of HepG2/ BHK-21 cells.

Table 1: Cytotoxicity of HepG2 cells and BHk-21 healthy cells upon FU and Se free- and nano-combinations.

\begin{tabular}{|c|c|c|c|c|}
\hline \multirow[t]{2}{*}{ Concentrations } & \multicolumn{2}{|c|}{ HepG2 cells $($ Mean \pm SD) } & \multicolumn{2}{|c|}{ BHK-21 cells $($ Mean \pm SD) } \\
\hline & $\mathbf{F U}+\mathrm{Se}$ & Nano-FU + Nano-Se & $\mathbf{F U}+\mathrm{Se}$ & Nano-FU + Nano-Se \\
\hline $0 \mu \mathrm{M} \mathrm{Se}+50 \mu \mathrm{M} \mathrm{FU}$ & $100 \pm 5$ & $100 \pm 5$ & $100 \pm 5$ & $100 \pm 5$ \\
\hline $2 \mu \mathrm{M} \mathrm{Se}+50 \mu \mathrm{M} \mathrm{FU}$ & $92.76 \pm 4.64$ & $78.95 \pm 3.94$ & $105 \pm \overline{5} .25$ & $118.33+5.97$ \\
\hline $4 \mu \mathrm{M} \mathrm{Se}+50 \mu \mathrm{M} \mathrm{FU}$ & $88.82+4.44$ & $75.00+3.75$ & $83.33+4.16$ & $166.66+8.33$ \\
\hline $6 \mu \mathrm{M} \mathrm{Se}+50 \mu \mathrm{M} \mathrm{FU}$ & $88.82 \pm 4.6$ & $71.05 \pm 3.52$ & $113.33 \pm 5.67$ & $135 \pm \overline{6} .75$ \\
\hline $8 \mu \mathrm{M} \mathrm{Se}+50 \mu \mathrm{M} \mathrm{FU}$ & $80.92 \pm 4.05$ & $69.08 \pm 3.43$ & $73.33 \pm 3.66$ & $108.33 \pm 5.41$ \\
\hline $10 \mu \mathrm{M} \mathrm{Se}+50 \mu \mathrm{M} \mathrm{FU}$ & $75+\overline{3} .75$ & $65.13+3.26$ & $108.33+5.47$ & $108.3+5.7$ \\
\hline $30 \mu \mathrm{M} \mathrm{Se}+50 \mu \mathrm{M} \mathrm{FU}$ & $75 \pm 3.75$ & $65.13 \pm 3.26$ & $90 \pm 4.5$ & $133.33 \pm 6.67$ \\
\hline $50 \mu \mathrm{M} \mathrm{Se}+50 \mu \mathrm{M} \mathrm{FU}$ & $71.05 \pm 3.55$ & $55.26 \pm 2.78$ & $61.66 \pm 3.08$ & $101.66 \pm 5.33$ \\
\hline
\end{tabular}

\section{FU and nano-FU induce HepG2 and BHK-21 cell death}

The cytotoxicities of FU and nano-FU were measured against hepatoma cell line (HepG2) and normal kidney cells (BHK-21) at different concentrations $(0,10,20,30,40$, and 50 $\mu \mathrm{M}$ ) using MTT assay. Data illustrated in (Fig. 4) shows the percentage of viability of HepG2/ BHK-21 cells after $24 \mathrm{~h}$ from treatments versus controls. The results revealed a dose dependent statistical decrease $(\mathrm{P}<0.05)$ in cell viability of HepG2/ BHK-21 cells. Moreover, there were no significant differences between both cell lines; where FU had the highest cytotoxic effects at 50 $\mu \mathrm{M}$ with $78.9 \%$ (Fig. 4a) and $76.0 \%$ (Fig. 4b) cell viabilities, respectively, while nano-FU had the highest cytotoxic effects at 50 $\mu \mathrm{M}$ with $82.8 \%$ (Fig. 4a) and $74.6 \%$ (Fig. 4b) cell viabilities, respectively, at $24 \mathrm{~h}$.

\section{Nano-FU plus nano-Se induce HepG2 cell death and BHK-21 cell proliferation}

The cytotoxicities of $50 \mu \mathrm{M}$ nano-FU and its free counterpart plus serial doses of nan-Se and its free counterpart (i.e. $0+0,50+2,50+4,50+6,50+8,50+10,50+30$, and $50+50 \mu \mathrm{M}$ ) were applied on HepG2 and BHk-21 cells and measured using MTT assay. Data illustrated in (Table 1) shows the percentage of HepG2/ BHK-21 cell viability after $24 \mathrm{~h}$ from treatments versus controls.

The results revealed a dose dependent statistical decrease ( $\mathrm{P}<0.01)$ in HepG2 cell viability. In case of HepG2 cell line, $\mathrm{FU}+\mathrm{Se}$ and nano- FU+ nano-Se had the highest cytotoxic effects at $50+50 \mu \mathrm{M}$ with $71.0 \%$ and $55.2 \%$ cell viabilities, respectively (Table 1).

In case of normal $\mathrm{BHK}-21$ cells, the $\mathrm{FU}+\mathrm{Se}$ results revealed a dose independent decrease $(\mathrm{P}<0.05)$ in cell viability. $\mathrm{FU}+\mathrm{Se}$ had the highest cytotoxic effects at $50+50 \mu \mathrm{M}$ with $61.6 \%$ cell viability (Table 1 ).

On the contrary, nano-FU+ nano-Se had no cytotoxic effects on BHK-21 cells, but this nano-formulation induces a strange cell proliferation pattern, reaching its maximum beak at 50 $\mu \mathrm{M}$ nano-FU plus $4 \mu \mathrm{M}$ nano-Se with $166.6 \%$ cell viability increment (Table 1). 
A]

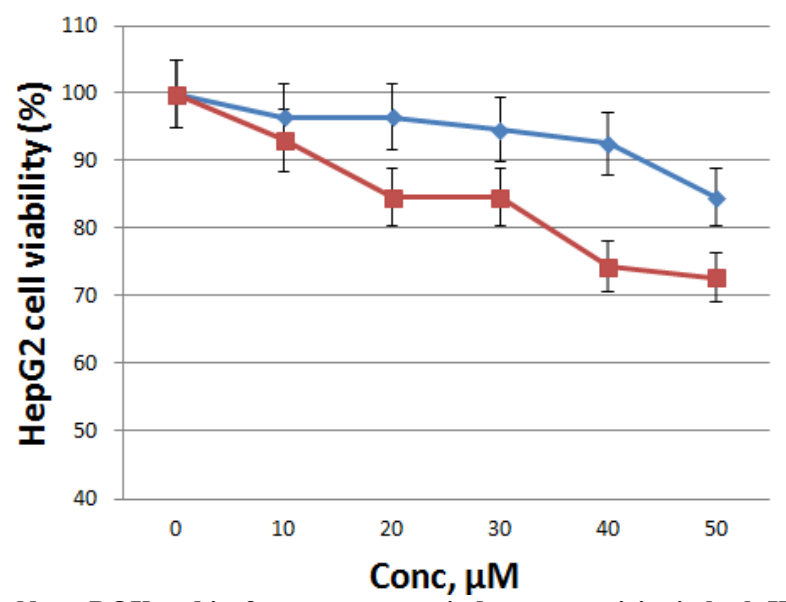

B]

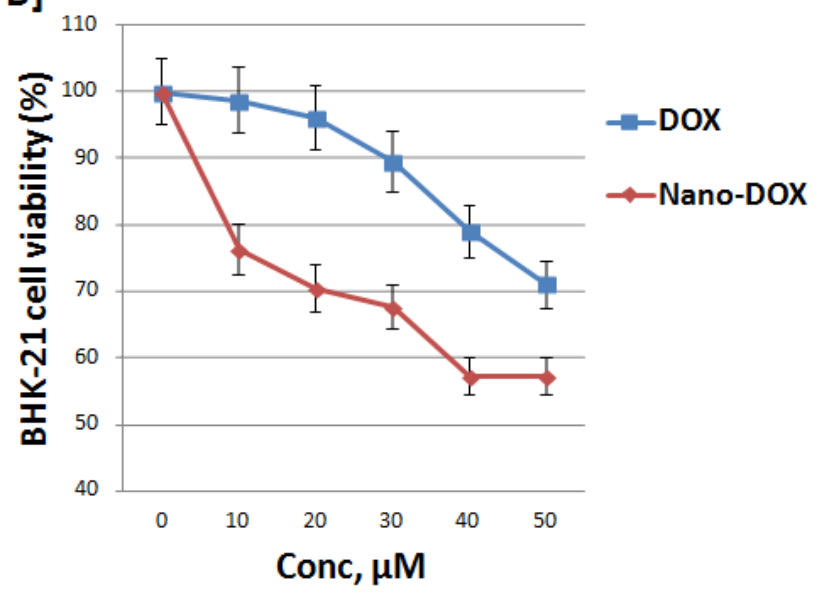

Fig. 5: Nano-DOX and its free counterpart induce cytotoxicity in both HepG2 (Fig. 5A) and BHk-21 (Fig. 5B) cells. Cytotoxicity of DOX and nano-DOX was evaluated by MTT assay using HepG2/BHk-21 cells. Absorbance was measured at 455 nm, using a microplate reader (BMG Labtech, Germany). The cell viability $(\%)$ was calculated and compared with the controls. Three independent runs $(\mathrm{n}=3)$ were performed for each experiment and represented as mean and standard deviation (SD) bars. The results revealed a dose dependent statistical decrease $(\mathrm{P}<0.05)$ in cell viability of HepG2/ BHK-21 cells.

Table 2: Cytotoxicity of HepG2 cells and BHk-21 healthy cells upon DOX and Se free- and nano-combinations.

\begin{tabular}{|c|c|c|c|c|}
\hline \multirow[t]{2}{*}{ Concentrations } & \multicolumn{2}{|c|}{ HepG2 cells $($ Mean + SD $)$} & \multicolumn{2}{|c|}{ BHK-21 cells $($ Mean + SD) } \\
\hline & $\mathrm{DOX}+\mathrm{Se}$ & Nano-DOX + Nano-Se & DOX+Se & Nano- DOX + Nano-Se \\
\hline $0 \mu \mathrm{M} \mathrm{Se}+50 \mu \mathrm{M}$ DOX & $100 \pm 5$ & $100 \pm 5$ & $100 \pm 5$ & $100 \pm 5$ \\
\hline $2 \mu \mathrm{M} \mathrm{Se}+50 \mu \mathrm{M} \mathrm{DOX}$ & $90.91 \pm 4.55$ & $84.87 \pm 4.21$ & $123.33 \pm 6.17$ & $186.66 \pm 9.33$ \\
\hline $4 \mu \mathrm{M} \mathrm{Se}+50 \mu \mathrm{M}$ DOX & $92.73 \pm 4.64$ & $82.89 \pm 4.37$ & $133.33 \pm 6.67$ & $206.66 \pm 10.32$ \\
\hline $6 \mu \mathrm{M} \mathrm{Se}+50 \mu \mathrm{M}$ DOX & $90.91+4.55$ & $80.92 \pm 4.04$ & $156.66 \pm 7.83$ & $180 \pm 9$ \\
\hline $8 \mu \mathrm{M} \mathrm{Se}+50 \mu \mathrm{M}$ DOX & $90.91 \pm 4.55$ & $75.00 \pm 3.75$ & $90 \pm \overline{4} .5$ & $190 \pm 9.5$ \\
\hline $10 \mu \mathrm{M} \mathrm{Se}+50 \mu \mathrm{M}$ DOX & $80.00 \pm 4.00$ & $65.13 \pm 3.79$ & $166.6 \overline{6} \pm 8.33$ & $210 \pm 10.5$ \\
\hline $30 \mu \mathrm{M} \mathrm{Se}+50 \mu \mathrm{M}$ DOX & $78.18 \pm \overline{3} .91$ & $63.19 \pm 3.15$ & $140 \pm 17$ & $183.3 \overline{3} \pm 9.17$ \\
\hline $50 \mu \mathrm{M} \mathrm{Se}+50 \mu \mathrm{M} \mathrm{DOX}$ & $67.27 \pm 3.36$ & $59.21 \pm 2.92$ & $176.66 \pm 11.81$ & $190 \pm \overline{9} .5$ \\
\hline
\end{tabular}

\section{DOX and nano-DOX induce HepG2 and BHK-21 cell death}

The cytotoxicities of DOX and nano-DOX were measured against hepatoma cell line (HepG2) and normal kidney cells (BHK-21) at different concentrations $(0,10,20,30,40$, and $50 \mu \mathrm{M}$ ) using MTT assay. Data illustrated in (Fig. 5) shows the percentage of viability of HepG2/ BHK-21 cells after $24 \mathrm{~h}$ from treatments versus controls. The results revealed a dose dependent statistical decrease $(\mathrm{P}<0.05)$ in cell viability of HepG2/ BHK-21 cells. Moreover, both DOX and nano-DOX show significant cell viability decreases in case of BHK-21 compared to HepG2 cell line; where DOX and nano-DOX had the highest cytotoxic effects at $50 \mu \mathrm{M}$ with $84.8 \%$ and $72.8 \%$ (Fig. 5) cell viabilities, respectively, while DOX and nano-DOX had the highest cytotoxic effects at $50 \mu \mathrm{M}$ with $71.0 \%$ and $57.1 \%$ cell viabilities, respectively, at $24 \mathrm{~h}$ (Fig. 5).

\section{DOX plus Se in nano- and free-forms induce HepG2 cell death and BHK-21 cell proliferation}

The cytotoxicities of $50 \mu \mathrm{M}$ nano-DOX and its free counterpart plus serial doses of nan-Se and its free counterpart (i.e. $0+0,50+2,50+4,50+6,50+8,50+10,50+30$, and $50+50 \mu \mathrm{M}$ ) were applied on HepG2 and BHk-21 cells and investigated using MTT assay. Data illustrated in (Table 2) shows the percentage of HepG2/ BHK-21 cell viability after $24 \mathrm{~h}$ from treatments versus controls. The results revealed a dose dependent statistical decrease $(\mathrm{P}<0.01)$ in HepG2 cell viability. In case of HepG2 cell line, $\mathrm{DOX}+\mathrm{Se}$ and nano-DOX+ nano-Se had the highest cytotoxic effects at $50+50 \mu \mathrm{M}$ with $67.2 \%$ and $42.4 \%$ cell viabilities, respectively, with a detectable IC50 equal to $20 \mu \mathrm{M}$ of nano-Se plus $50 \mu \mathrm{M}$ of nano-DOX (Table 2). In case of normal BHK-21 cells, the results revealed significant dose increase $(\mathrm{P}<0.05)$ in cell viability. DOX+Se had no cytotoxic effects, but a proliferative effect on normal BHK-21 cells, reaching its maximum beak at 50 $\mu \mathrm{M}$ nano-DOX plus $50 \mu \mathrm{M}$ nano-Se with $176.6 \%$ cell viability increment (Table 2), while there was a drop in this increment at 50 $\mu \mathrm{M}$ nano-DOX plus $8 \mu \mathrm{M}$ nano-Se with $90 \%$ cell viability (Table 2). On the other side, nano-DOX +nano-DOX had no cytotoxic effects on BHK-21 cells, but this nano-formulation induces a strange consistent cell proliferation pattern, reaching its maximum beak and further stabilized from adding the lowest to highest dose of nano-Se plus $50 \mu \mathrm{M}$ nano-DOX with around $200 \%$ cell viability increment (Table 2). 
Table 3: Cell cycle analyses upon therapies with individual nano-Se or combinated with nano-DOX compared to control.


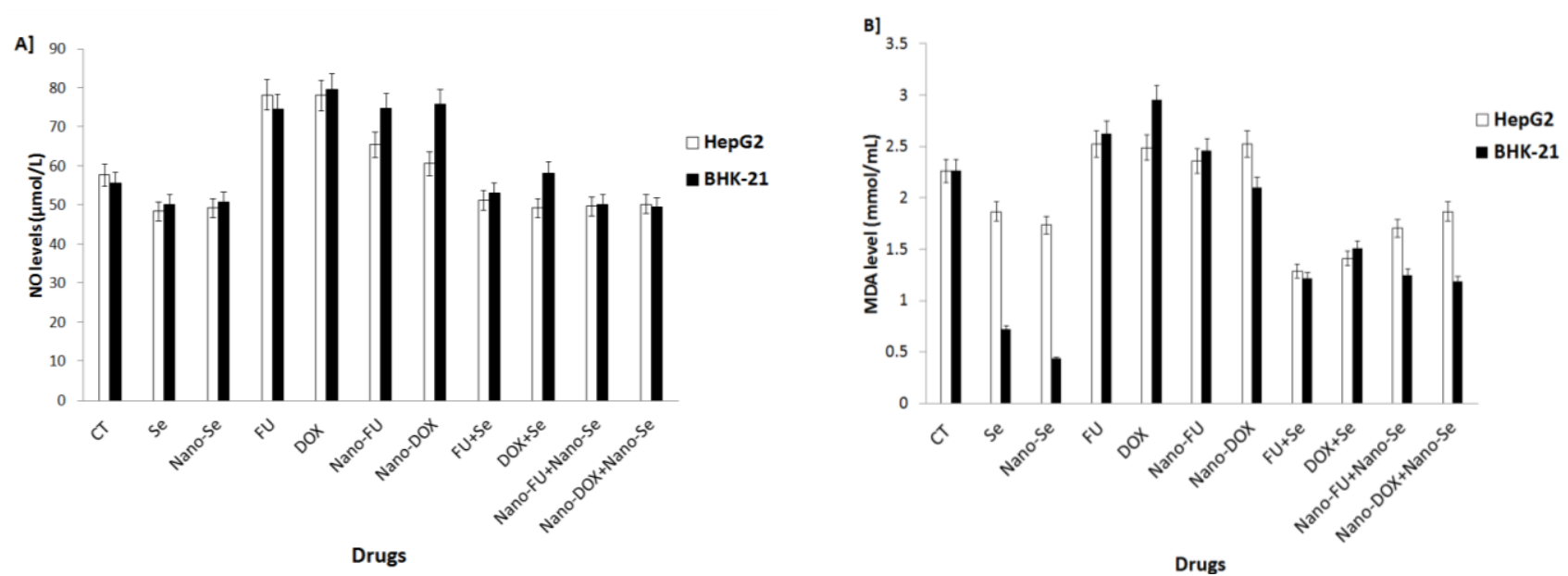

Fig. 6: NO (Fig. 6A) and MDA (Fig. 6B) levels were measured in HepG2 and BHK-21 cells up on Se, FU, and DOX in nano-, free-, and combinatorialformulations. Different nano-treatments and their free counterparts were added in the media. $10 \mu \mathrm{M}$ of all Se formulations plus $50 \mu \mathrm{M}$ of all DOX and $5 \mathrm{FU}$ formulations were used in this experiment. A) Griess reagent was used to converts nitrite to a deep purple azo compound. The amount of the azo chromophore accurately reflected nitric oxide amount in the samples. Optical density was measured at OD $540 \mathrm{~nm}$. B) The free MDA present in the sample was reacted with Thiobarbituric Acid (TBA) to generate a MDA-TBA adduct, which was quantified at OD $532 \mathrm{~nm}$ using the microplate reader (BMG Labtech, Germany). Three independent runs $(\mathrm{n}=3)$ were performed for each experiment and represented as mean and standard deviation (SD) bars. Oxidative markers were significantly statistical decreased $(\mathrm{P}<0.05)$ upon Se and nano-Se treatments compared to control, while MDA was significant abolished $(\mathrm{P}<0.01)$ up on BHK-21 treatment with Se and nano-Se. Then, their levels were lucidity statistical elevated $(\mathrm{P}<0.05)$ up on FU and DOX nano- and free- formulations compared to control.

\section{Nano-DOX plus nano-Se induce HepG2 cell cycle arresting, while sparing BHK-21 cell proliferation}

Table 3 show that HepG2 cells were arrested and accumulated in G0/G1 phase under the effect of $10 \mu \mathrm{M}$ of NanoSe $(73.4 \%)$ and $10 \mu \mathrm{M}$ of Nano-Se plus $50 \mu \mathrm{M}$ of nano-DOX (73.5\%) compared to control $(65.9 \%)$. This has reduced the flow of cells directed toward both $\mathrm{S}$ and/or G2/M phases led to decrease in the frequency $(\%)$ of those cells at these stations $(17.1 \%$ and $15.1 \%$, respectively) compared to control (22.9\%). However, the frequency $(\%)$ of HepG2 cells was decreased in S phase up on 10 $\mu \mathrm{M}$ of Nano-Se plus $50 \mu \mathrm{M}$ of nano-DOX combinatorial treatment compared to control and even $10 \mu \mathrm{M}$ of Nano-Se. On the contrary, the percentage of normal BHK-21 cells were increased at G2/M up on treatment with $10 \mu \mathrm{M}$ of Nano-Se plus $50 \mu \mathrm{M}$ of nano-DOX (24.1\%) after decreasing up on treatment with $10 \mu \mathrm{M}$ of Nano-Se $(17.7 \%)$ compared to control $(21.3 \%)$.

\section{Se formulations induce NO/MDA re-homeostasis when combined with DOX/FU formulations to avoid harming BHK- 21 cells}

Fig. 6 shows the MDA and NO levels produced by HepG2 and BHK-21 cells up on treatments versus controls; where both oxidative markers were significantly statistical decreased $(\mathrm{P}<$ 0.05) up on Se and nano-Se treatments compared to control, while MDA was significant abolished $(\mathrm{P}<0.01)$ up on BHK-21 treatment with $\mathrm{Se}$ and nano-Se. Then, their levels were lucidity statistical elevated ( $\mathrm{P}<0.05)$ up on FU and DOX nano- and freeformulations compared to control. Intriguingly, the combination of both Se and DOX/FU formulations re-balance the MDA and NO levels in HepG2 and BHK-21 cells to avoid the harmful effects of over-stress on the normal BHK-21 cells, making this therapeutic combinatorial formula specific to kill liver cancer HepG2 cells, while sparing BHK-21 cells healthy.

\section{Slight bioenergetic deprivation through glycolytic inhibition}

Fig. 7 illustrates the accumulated glucose in the RPMI1640 media over HepG2 cells and hanks' MEM media over BHK21 cells post-therapy compared to control after $24 \mathrm{~h}$ incubation with the tested nano-drugs and their free counterparts. Briefly, the glucose transports in HepG2 and BHK-21 control cells were working well and up-taking glucose from the media over $24 \mathrm{~h}$, resulting in a significant decrease in the glucose levels in the media over the cells $(\mathrm{P}<0.01)$. After that, the transports got slight inhibition by the nano-drugs and their free counterparts, resulting 
in decreasing the ability of uptaking glucose from the media over $24 \mathrm{~h}$ with drug incubations, and in turn increasing the levels of glucose in the media compared to the $24 \mathrm{~h}$-control.



Fig. 7: Glucose levels in media over HepG2 and BHK-21 cell lines after treatment with Se, FU, and DOX in nano-, free-, and combinatorialformulations. A statistical significant decrease in the glucose levels in the media over the cells $(\mathrm{P}<0.01)$.

\section{Individual and combinatorial Se formulations crosstalk with Zn to kill cancer cells}

Fig. 8 shows that $\mathrm{Zn}$ levels were significantly increased up on HepG2 and BHK-21 cell treatments with any Se-based drug; $\mathrm{Se}$, nano-Se, $\mathrm{FU}+\mathrm{Se}, \mathrm{DOX}+\mathrm{Se}$, nano-FU+ nano-Se, and nano$\mathrm{DOX}+$ nano-Se with different ratios, while there were no significant changes up on FU, DOX, nano-FU, and nano-DOX therapies. This represents that Se formulations crosstalk with $\mathrm{Zn}$ to kill cancer HepG2 cells, but up to date the exact mechanism of action is still obscured. Targeting cancer cells, while sparing normal cells with minimal cytotoxic effect, is becoming one of the most important stumbling blocks cancer therapies. Cell death mechanism may be resulted in up-regulation of pro-apoptotic members and down-regulation of cancer metabolism (Pan et al., 2013; Liu et al., 2015). Nanodrug delivery systems were widely used by improving the stimuli-triggered drug release and cancertargeted drug delivery to minimize the adverse events (He et al., 2014; Liu et al., 2015). Selenium nanoformulations become under the focus as nanocarriers and a potential chemotherapeutic agent due to their biocompatibility straight forward synthesis, lowtoxicity, degradability in-vivo, excellent antioxidant activity and chemo-preventative effects. In addition, nanoparticles can be used as 5-fluorouracil (5-FU) and doxorubicin (DOX) carriers (Huang et al., 2013; Liu et al., 2015). To overcome the instability problem of Se nanoformulations, we used 5FU/DOX PLGA-CS-PEG nanoformulation in combination. Chitosan-based nanoformulation had a powerful function in stabilizing Se nanoformulations and stimulating significant apoptosis in cancer cells (Feng et al., 2014). Scientists also found that the force of $\mathrm{Se}-\mathrm{O}$ and $\mathrm{Se}-\mathrm{N}$ bonds leads to the stable structure of the conjugates between 5-FU and NPs (Feng et al., 2014). Also, using Se nanoformulations as a drug carrier for FU/DOX (FU/DOX- NPs) exhibited a broad spectrum of growth inhibition against human cancer cells besides overlapping on the multidrug resistance problem (Liu et al., 2015), which supports our results.

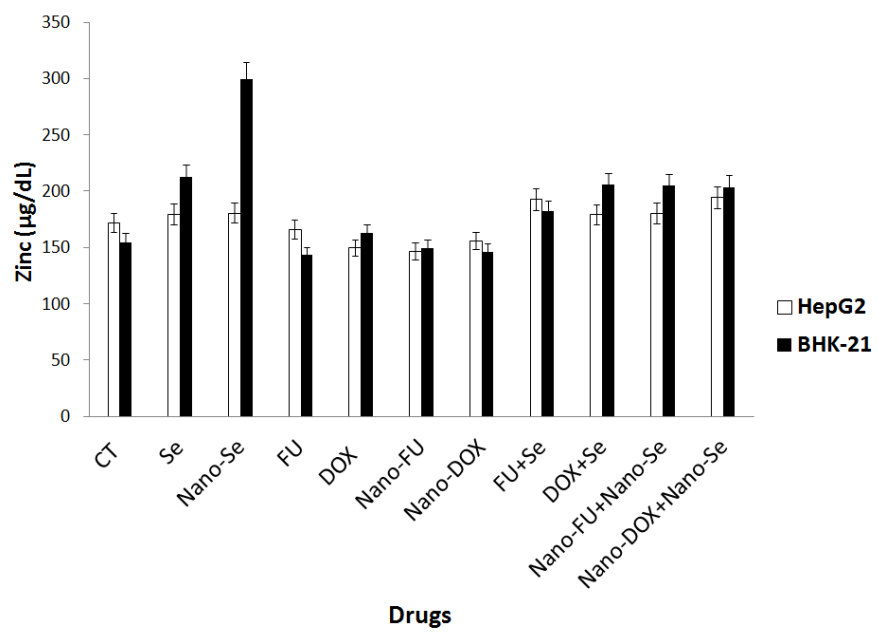

Fig. 8: Zinc levels in media over HepG2 and BHK-21 cell lines after treatment with Se, FU, and DOX in nano-, free-, and combinatorial- formulations. Different nano-treatments and their free counterparts were added in the media $10 \mu \mathrm{M}$ of all Se formulations plus $50 \mu \mathrm{M}$ of all DOX and 5FU formulations were used in this experiment. Three independent runs $(n=3)$ were performed for each experiment and represented as mean and standard deviation (SD) bars.

In our study, Se nanoformulation in combination with FU/DOX nanoformulations has been proposed as a potential chemotherapeutic agent due to its low toxicity and its ability to arrest the cell cycle of cancer cells. Our results confirm the cell cycle arrest at the S phase, where others declared the depletion of the cell population in $\mathrm{G} 0 / \mathrm{G} 1$ phase with increasing in $\mathrm{S}-\mathrm{G} 2 / \mathrm{M}$ (Lopez-Heras et al., 2014). Selenium (Se) either in the molecular size or nano-size has miracle anti-neoplastic properties due to several pathways, inducing apoptosis by MAPK and AKT pathways, decreasing the speed of tumor proliferation, or increasing the speed of apoptosis through Akt/Mdm2/AR pathways (Feng et al., 2014; Liu et al., 2015). Cancer cells have a predominant glycolytic metabolism, even under aerobic conditions (Cuezua et al., 2009). The energetic alteration of cancer cells by inhibiting glucose uptaking proposes a potential target for cancer therapy (Cuezua et al., 2009). In some cancers, this glycolytic phenotype is accompanied by a loss of bioenergetic activity in mitochondria (lopez-Rios et al., 2007), which can be used as bioenergetic signatures for cancer progression and eradication (Sanchez-Argo et al., 2010). Recent findings indicated that the bioenergetic signature represents a functional index of metabolic activity because it correlates with the rate of glucose utilization by cancer cells (Sanchez-Argo et al., 2010). This is in agreement with the current results concerning glucose levels which indicate that the used nanoformulations reduce the glucose uptake through cancer cells. Selenium administration raises the level of Seleniumbinding protein 1 (SBP1, SELENBP1). In SBP1, Se is strongly linked with the peptide instead of incorporated co-translationally 
as glutathione peroxidase 1 (GPx1). The expression of SBP1is observed in different cells and tissues (liver, heart, lung, and kidney) and positioned in the nucleus and/or cytoplasm depending on cell type, degree of differentiation, and environmental stimuli (Ying et al., 2015). There are a correlation between SBP1 level and changes of lipid/glucose metabolism-related proteins (GAA, GAPDH, ALDH2, Thioredoxin, ENO3, UGDH and Lumican) through the implication of multiple signaling axis such as MAPK/Wnt, DKK1, ANXA4 or NF-kB, because any one of these molecules plays pivotal role in carcinogenesis and progression through controlling downstream targets (Ying et al., 2015). UGDH (UDP-glucose dehydrogenase) catalyzes oxidation of UDPglucose to yield UDP-glucuronic acid, a precursor of hyaluronic acid (HA) and other glycosaminoglycans (GAGs) in extracellular matrix. UGDH deficiency suppresses cell aggregation and migration (Wang et al., 2010). Furthermore, ALDH2 inactivation enhances cytotoxicity induced by lipid peroxidation (Niknahad $e t$ al., 2003). The antioxidant effect of $\mathrm{Se}$ is imputed to selenoenzymes, the glutathione peroxidase family (GPXs) and thioredoxin reductase (TR). The GPXs function to detoxify many peroxides, likes $\mathrm{H}_{2} \mathrm{O}_{2}$, fatty acids hydroperoxides, phospholipid hydroperoxide and hydroperoxy groups of thymine (Zhang et al., 2001). Also, TR plays multiple roles in conjunction with its substrate thioredoxin in detoxification reaction (Zhang et al., 2001). In addition, Se has pro-oxidant catalytic activity through its reaction with thiols producing superoxide $\left(\mathrm{O}^{-2}\right)$, hydrogen peroxide and other cascading oxyradicals (Shen et al., 2001; Zhang et al., 2001). Se nanoformulations have biological activity similar to those of Se on Se-dependent enzyme biosynthesis but with much lower acute toxicity (Zhang et al., 2001). The previous observations proved our output indicated that Se nanoformulations detoxify NO and MDA that may be induced after the application of Fu/Dox- nanoformulations. There are a strong relation between Se and zinc for tackling cancer cells. Xiao et al. (2012) reported that the depletion of Se and zinc levels promote the growth of cancer cells. Both of Se and zinc antagonize oxidative stress, induce apoptosis, and arrest cell cycle (Yu et al., 2006). These results match those in ours which indicated that Se application leads to zinc increment, which in turn leads to cancer eradication based on cytotoxicity and zinc analysis results. The present findings justify the potential of Se, Se nanoformulations and/or FU/DOX- nanoformulations for tackeling hepatocellular carcinoma through targeting redox mode and reducing glucose consumption beside their pro-apoptotic activities. Also, using Se nanoformulations as a drug carrier in addition to FU/DOX nanoformulations enhances their chemopreventive effects against liver cancer. This opens a wide window of efficacious pro-drugs against HCC and provides low toxic doses versus normal cells.

Conflict of Interests: There are no conflicts of interest.

\section{REFERENCES}

Abdel-Atti E. HCC Burden in Egypt. Gastroenterol Hepatol Open Access, 2015; 2(3): 00045.
Abd-Rabou AA, Ahmed HH. CS-PEG decorated PLGA nanoprototype for delivery of bioactive compounds: A novel approach for induction of apoptosis in HePG2 cell line. Advances in Medical Sciences. 2017; In Press.

Amiji M M. Surface modification of chitosan membranes by complexation- interpenetration of anionic polysaccharides for improved blood compatibility in hemodialysis. Journal of Biomaterials Science, Polymer Edition, 1997; 8(4): 281-298.

Cuezva JM, Ortega AD, Willers I, Sánchez-Cenizo L, Aldea M, Sánchez-Aragó M. The tumor suppressor function of mitochondria: translation into the clinics. Biochim Biophys Acta, 2009; 1792(12):11451158.

Feng Y, Su J, Zhao Z, Zheng W, Wu H, Zhang Y, Chen T. Differential effects of amino acid surface decoration on the anticancer efficacy of selenium nanoparticles. Dalton Trans, 2014; 43(4):1854-1861.

Gomaa AI, Hashim MS, Waked I. Comparing staging systems for predicting prognosis and survival in patients with hepatocellular carcinoma in Egypt. PLoS One, 2014; 9: e90929.

Gref R, Minamitake Y, Peracchia MT, Trubetskoy V, Torchilin $\mathrm{V}$, Langer R. Biodegradable long-circulating polymeric nanospheres. Science, 1994; 263:1600- 1603.

He L, Huang Y, Zhu H, Pang G, Zheng W, Wong YS, Chen T. Cancer-targeted monodisperse mesoporous silica nanoparticles as carrier of ruthenium polypyridyl complexes to enhance theranostic effects. Adv Funct Mater, 2014; 24 (19): 2754-2763.

Hu X, Zhou J, Zhang N, Tan H, Gao C. Preparation and properties of an injectable scaffold of poly(lactic-co-glycolic acid) microparticles/chitosan hydrogel. J Mech Behav Biomed Mater, 2008; 1(4):352-359.

Huang Y, He L, Liu W, Fan C, Zheng W, Wong Y-S, Chen T. Selective cellular uptake and induction of apoptosis of cancer-targeted selenium nanoparticles. Biomaterials, 2013; 34:7106-7116.

Illum L. Chitosan and its use as a pharmaceutical excipient. Pharm Res, 1998; 15(9):1326-1331.

Liao W, Yu Z, Lin Z, Lei Z, Ning Z, Regenstein JM, Yang J, Ren J. Biofunctionalization of Selenium Nanoparticle with Dictyophora Indusiata Polysaccharide and Its Antiproliferative Activity through DeathReceptor and Mitochondria-Mediated Apoptotic Pathways. Sci Rep, 2015;5:18629.

Liu T, Zeng L, Jiang W, Fu Y, Zheng W, Chen T. Rational design of cancer-targeted selenium nanoparticles to antagonize multidrug resistance in cancer cells. Nanomedicine. 2015; 11(4):947-9458.

Lopez-Heras I, Sanchez-Diaz R, Anunciação DS, Madrid Y, Luque-Garcia JL, Camara C. Effect of chitosan-stabilized selenium nanoparticles on cell cycle arrest and invasiveness in hepatocarcinoma cells revealed by quantitative proteomics. J Nanomed Nanotechnol, 2014; 5:5.

López-Ríos F, Sánchez-Aragó M, García-García E, Ortega AD, Berrendero JR, Pozo-Rodríguez F, López-Encuentra A, Ballestín C, Cuezva JM. Loss of the mitochondrial bioenergetic capacity underlies the glucose avidity of carcinomas. Cancer Res. 2007; 67(19):9013-9017.

Magistri P, Tarantino G, Ballarin R, Berretta M, Pecchi A, Ramacciato G, DI Benedetto F. The Evolving Role of Local Treatments for HCC in the Third Millennium. Anticancer Res, 2017; 37(2):389-401.

Holah NS, El-Azab DS, Aiad HA, Sweed DM. Hepatocellular carcinoma in Egypt: epidemiological and histopathological properties. Menoufia Medical Journal, 2015; 28:718-724.

Niknahad H, Siraki AG, Shuhendler A, Khan S, Teng S, Galati G, Easson E, Poon R, O'Brien PJ. Modulating carbonyl cytotoxicity in intact rat hepatocytes by inhibiting carbonyl-metabolizing enzymes. I. Aliphatic alkenals. Chemico-Biological Interactions, 2003; 143-144: 107117.

Pan L, Liu J, He Q, Wang L, Shi J. Overcoming multidrug resistance of cancer cells by direct intranuclear drug delivery using TATconjugated mesoporous silica nanoparticles. Biomaterials, 2013; 34:27192730 .

Parveen S, Mitra M, Krishnakumar S, Sahoo SK. Enhanced antiproliferative activity of carboplatin-loaded chitosan-alginate 
nanoparticles in a retinoblastoma cell line. Acta Biomater, 2010; 6(8):3120-31.

Sánchez-Aragó M, Chamorro M, Cuezva JM. Selection of cancer cells with repressed mitochondria triggers colon cancer progression. Carcinogenesis, 2010; 31(4):567-576.

Shen HM, Yang CF, Ding WX, Liu J, Ong CN. Selenite-treated HepG2 cells: mitochondria serve as the main target, Free Rad. Biol. Med, 2001; 30: 9-12.

Wang G, Guo Y, Yang G, Yang L, Ma X, Wang K, Zhu L, Sun J, Wang $X$, Zhang $\mathrm{H}$, Mitochondria-Mediated Protein Regulation Mechanism of Polymorphs-Dependent Inhibition of Nanoselenium on Cancer Cells. Sci Rep, 2016; 6:31427.

Wang TP, Pan YR, Fu CY, Chang HY. Down-regulation of UDP-glucose dehydrogenase affects glycosaminoglycans synthesis and motility in HCT-8 colorectal carcinoma cells. Exp Cell Res, 2010; 316: 2893-2902.

Xiao H, Jiang Y, Qi Y, Zhou X, Gong C, Huang C, Li M. Effects of selenium and zinc on the proliferation of human esophageal cancer cell line studied by serophysiology. Wei Sheng Yan Jiu, 2012; 41(2):185-190.

Yau T, Chan P, Epstein R, Poon RT. Evolution of systemic therapy of advanced hepatocellular carcinoma. World J Gastroenterol, 2008; 14(42):6437-41.
Ying Q, Ansong E, Diamond AM, Lu Z, Yang W, Bie X. Quantitative proteomic analysis reveals that anti-cancer effects of selenium-binding protein 1 in vivo are associated with metabolic pathways. PLoS One, 2015; 10(5):e0126285.

Yu RA, Xia T, Wang AG, Chen XM. Effects of selenium and zinc on renal oxidative stress and apoptosis induced by fluoride in rats. Biomed Environ Sci, 2006; 19(6):439-444.

Liang Y, Zhou Y, S, Chen T. Microwave-Assisted Syntheses of Benzimidazole-Containing Selenadiazole Derivatives That Induce CellCycle Arrest and Apoptosis in Human Breast Cancer Cells by Activation of the ROS/AKT Pathway. Chem Med Chem, 2016; 11: 2339 - 2346.

Zhang JS, Gao XY, Zhang LD, Bao YP. Biological effects of a nano red elemental selenium. Biofactors, 2001; 15(1):27-38.

\section{How to cite this article:}

Shalby AB, Abd-Rabou AA, Ahmed HH. Nano-Se crosstalks with nano-DOX/FU to selectively hack hepatic cancer cells and spare normal cells healthy: A mechanism-based study. J App Pharm Sci, 2017; 7 (08): 003-012. 